\title{
TLR2 and IL 18 in Obese Diabetic Patient
}

Zakia AZ Mohamed ${ }^{1}$, Sabah E Abd-Elraheem ${ }^{1}$, Eslam MA El- Nahrery ${ }^{4}$, Seham Sabry ${ }^{2}$ and Maha Salah Eldin Mohamed ${ }^{3}$

${ }^{1}$ Departments of Clinical Pathology, Al-Azhar University, Cairo, Egypt

${ }^{2}$ Internal Medicine, Physical Medicine, Al-Azhar University, Cairo, Egypt

${ }^{3}$ Rheumatology and Rehabilitation, Al-Azhar University, Cairo, Egypt

${ }^{4}$ Chemistry-Biochemistry Branch, Suez University, Egypt

*Corresponding author: El-Nahrery E, Chemistry-Biochemistry Branch, Faculty of Science, Suez University, Egypt, Tel: 01155941100; E-mail: eslam_elnahrery2001@yahoo.com

Rec date: April 16, 2016; Acc date: May 24, 2016; Pub date: May 29, 2016

Copyright: (c) 2016 Mohamed ZAZ, et al. This is an open-access article distributed under the terms of the Creative Commons Attribution License, which permits unrestricted use, distribution, and reproduction in any medium, provided the original author and source are credited.

\begin{abstract}
Objective: This study was conducted to determine expression ofTLR2 in peripheral blood monocytes and the proinflammatory cytokine IL18 in type 2 diabetic obese patients to explain a possible pathophysiological link between obesity and inflammation leading to insulin resistance.

Methodology: This study was conducted in Al-Zahra University Hospital .The study population consisted of 40 patients divided into 3 groups : 15 obese non diabetic patients, 15 diabetic obese patients and 10 normal body weight clinically and laboratory free served as a control group. All studied groups were subjected to clinical examination and laboratory investigations which include lipid profile, fasting blood glucose, $\mathrm{Hb} A 1 \mathrm{C}$, serum insulin, HOMA IR, Serum IL 18 and flow cytometric analysis expression of TLR-2 on CD14+ monocytes.
\end{abstract}

Results: IL-18 levels were significantly higher in obese non diabetic group(61.5 $\pm 8.2 \mathrm{ng} / \mathrm{l})$ and diabetic obese group $(62.8 \pm 10.9 \mathrm{ng} / \mathrm{l})$ when compare to normal control group $(26.5 \pm 6.5 \mathrm{ng} / \mathrm{l})(\mathrm{p}<0.000)$, while there were no significant change between obese non diabetic group and diabetic obese group regarding IL-18 levels ( $p$. 713 ) and there was a highly significant increase in TLR-2 MFI in obese non diabetic group $(9.3+3.2)$, diabetic obese group $(16.7+3.4)$ when compared to controls group $(4.5+1.2)$, also levels of TLR-2MFI were significantly higher in obese diabetic group compared to obese non diabetic group $(p<0.001)$. There was a significant increase in HOMA-IR in obese non diabetic group $(3.81+0.97)$, diabetic obese group $(11.55+3.89)$ when compared to controls group $(2.46+$ $0.95)(p<0.0025)(P<0.001)$. Also levels of HOMA IR were significantly higher in obese diabetic group compared to obese non diabetic group $(p<0.00)$. There was a significant increase in insulin levels in obese non diabetic group $(16.4+3.72 \mathrm{uU} / \mathrm{L})$, diabetic obese group $(21.40+3.98 \mathrm{uU} / \mathrm{l})$ when compared to controls group $(11.2+2.94 \mathrm{uU} / \mathrm{L})(\mathrm{p}<$ 0.008 ), also levels of insulin were significantly higher in obese non diabetic group compared to obese diabetic group $(p<0.0014)$. There was a significant positive correlation between TLR2 MFI and each of insulin, IL18 and BMI in obese non diabetic as well as diabetic obese patients.

Conclusions: This study demonstrated that the expression of TLR-2 on CD14+ monocytes was up regulated in obese non diabetic and obese diabetic patients and was associated with inflammatory response as assessed by increased serum levels of IL-18.

Keywords: Obesity; Inflammation; Toll like receptor-2; IL-18

\section{Introduction}

Obesity is a worldwide epidemic, and approximately 700 million adults were obese in 2015. Obesity is a major risk factor for the development of metabolic syndrome (MetS) and other associated health complications $[1,2]$.

There is a close association between obesity and type 2 diabetes. The likelihood and severity of type 2 diabetes are closely linked with body mass index (BMI). There is a seven times greater risk of diabetes in obese people compared to those with normal healthy weight, with a threefold increase in risk for overweight people. Whilst it is known that body fat distribution is an important determinant of increased risk of diabetes, the precise mechanism of association remains unclear. It is also uncertain why not all people who are obese develop type 2 diabetes and why not all people with type 2 diabetes are obese $[3,4]$.

Type 2 diabetes constitutes a group of metabolic aberrations including hyperglycemia, inflammation, and insulin resistance that increase the risk of cardiovascular disease. There appears to be a strong correlation between insulin resistance and low-grade inflammation $[5,6]$.

The mechanisms by which metabolic abnormalities cause cardiovascular disease are not clear. Considerable evidence indicates that the harmful effects of elevated glucose are mediated by receptors leading to increased inflammation and oxidative stress [7].

The associations between elevated levels of circulating C-reactive protein, proinflammatory cytokines, and homeostasis model assessment-insulin resistance (HOMA-IR) in diabetic patients suggest that inflammation is an important etiological factor in the 
development of both insulin resistance and type 2 diabetes. Moreover, inflammatory markers link the pathology of insulin resistance and type 2 diabetes. Activation of the innate immune system via toll-like receptors (TLRs) is implicated in the pathogenesis of insulin resistance, diabetes, and atherosclerosis [8].

Complementary genetic studies link TLR2 and TLR4 polymorphisms to type 2 diabetes, suggesting a causal relationship between TLR function and diabetes and its complications [9].

Most TLRs are on the cell surface, except for TLR3,-7,-8, and -9 , which reside mainly in the endosomes. TLRs initiate an immune response after recognition of specific ligands such as pathogenassociated molecular patterns (PAMPs) derived from pathogens as well as tissue damage and inflammation-induced no microbe danger signals such as damage-associated molecular patterns (DAMPs) Various endogenous ligands, such as saturated fatty acids (SFAs), modified low-density lipoproteins (LDLs), heat shock proteins (HSPs), high-mobility group box 1 (HMGB1), extracellular matrix degradation products, and advanced glaciation end-products, act as DAMPs and are recognized by TLRs, especially TLR 2 or TLR4, triggering a proinflammatory response [10].

IL-18 is a member of the IL- 1 family of cytokines and was originally described as an interferon gamma (IFN- $\gamma$ ) inducing factor. The cytokine is produced by macrophages, endothelial cells, vascular smooth muscle cells, dendritic cells and Kuepfer cells. IL-18 is also produced in adipocytes, but non-adipocyte cells have been identified as the main source of IL-18 in adipose tissue [11].

IL-18 is a potent proinflammatory cytokine which enhances $\mathrm{T}$ cell and natural killer cell maturation, as well as the production of cytokines, chemokines and cell adhesion molecules [12].

Interestingly, a study showed that one such polymorphism was associated with increased serum levels of IL-18, impaired insulin sensitivity and increased risk of having the metabolic syndrome, suggesting that IL-18 might be involved in the pathogenesis of the syndrome [13].

\section{Aim of the Work}

This study was conducted to determine expression of TLR2 in peripheral blood monocytes and the proinflammatory cytokine IL18 in type 2 obese diabetic patients to explain a possible pathophysiological link between obesity and inflammation leading to insulin resistance.

\section{Subject and Methods}

This study was conducted in Al-Zahra University Hospital in Cairo. The study population consisted of 40 patients divided into 3 groups : 15 obese non diabetic patients, 15 diabetic obese patients and 10 normal body weight clinically and laboratory free served as a control group.

Exclusion criteria included myocardial infarction, congestive heart failure, malignancy, hepatic cirrhosis, end-stage renal disease and hypertension.

Patients were divided into 2 groups: Obese non-diabetic patients, they were ( 5 males, 10 females), their ages ranged from $38-53$ years old with a mean of $(45+7.9)$. Obese diabetic patients, they were ( 6 males, 9 females) their ages ranged from 39-51 years old with a mean of (45.0 \pm 5.7). Healthy control: 15 normal body weight clinically and laboratory free served as a control group ( 6 males, 9 females), their ages ranged from 30-46 years old with a mean of $(40.8+5.7)$. Contributors completed a medical history; physical examinations, including respiratory, cardiac and other system examinations were done. Body mass index (BMI) was calculated using the equation BMI = weight (inkilograms) divided by the square of the height (in meters).

\section{All patients were subjected to the following}

- Full history and clinical examination.

- Laboratory investigations: Six $\mathrm{ml}$ of fasting venous blood samples was taken from each subject participating in the study and divided into two portions as follows:

- Three ml collected into EDTA containing tube for estimation of TLR 2 expression on peripheral blood monocytes by flow cytometry and $\mathrm{Hb} \mathrm{AlC}$ was measured by colorimetric method (Gonen and Rubenstein 1978) using kits provided by stanbio laboratory, Texas, USA.

- Three ml collected into a plan tube, left to clot then centrifuged at $1600 \mathrm{rpm}$ for 20 minutes and serum was separated and used for estimation of: Lipid profile and fasting blood glucose were done onHitachi911auto-analyzer (Roche-Hitachi, Japan).

- Serum insulin was measured by using Enzyme quantitative immunoassay using DRG Kit GmbH, Germany on ELISA system (Reader A3 1851, Washer 909) FROM DAS (ITALY).

HOMA index $\geq 2.7$ is considered as insulin resistance [14].

Serum IL-18 assay was performed using ELISA Assay Glory Science Co., Ltd. Del Rio, TX 78840, USA. Briefly, this assay employs a quantitative sandwich enzyme immunoassay technique that measures IL-18.

\begin{tabular}{|c|c|c|c|}
\hline \multirow{3}{*}{ Variables } & $\begin{array}{l}\text { Obese non } \\
\text { diabetic }\end{array}$ & Diabetic obese & Control \\
\hline & $N=15$ & $N=15$ & $N=10$ \\
\hline & \multicolumn{3}{|l|}{ Mean \pm SD } \\
\hline Age (year) & $45 \pm 7.9$ & $45.0 \pm 5.7$ & $37.8 \pm 7.7$ \\
\hline BMI & $39.9 \pm 8.06$ & $34 \pm 6.3$ & $22.2 \pm 3.9$ \\
\hline $\mathrm{FBS}(\mathrm{mg} / \mathrm{dl})$ & $94.7 \pm 8.06$ & $214 \pm 32$ & $89.4 \pm 10.74$ \\
\hline TG (mg/dl) & $105.4 \pm 34.5$ & $165.6 \pm 77.4$ & $80.4 \pm 24.9$ \\
\hline Chol & $201.5 \pm 38.57$ & $180.4 \pm 34.5$ & $112.1 \pm 16.1$ \\
\hline HOMA-IR & $3.81 \pm 0.97$ & $11.55 \pm 3.89$ & $2.46 \pm 0.95$ \\
\hline Insulin $(\mu \mathrm{IU} / \mathrm{ml})$ & $16.4 \pm 3.72$ & $21.40 \pm 3.98$ & $11.2 \pm 2.94$ \\
\hline IL-18 (ng/l) & $61.5 \pm 8.2$ & $62.8 \pm 10.9$ & $26.5 \pm 6.5$ \\
\hline TLR2 (MIF) & $9.3 \pm 3.2$ & $16.7 \pm 3.4$ & $4.5 \pm 1.2$ \\
\hline
\end{tabular}

Table 1: Demographic, clinical and laboratory of normal controls, obese diabetic and obese patients

Flow cytometric analysis of expression of TLR2 on CD14+ monocytes: Flow cytometric analysis was performed in fresh samples within 1 hour of blood collection, 100 microliters of EDTA anti coagulated blood was stained with combination of 10 microliters conjugated phycoerythrin (PE) labeled monoclonal antibodies for human TLR2 and 10 microliters conjugated fluorescence 
isothiocyanate. (FITC) labeled monoclonal antibodies for CD14. The mixture was incubated in dark room for 30 minutes then $1 \mathrm{ml}$ of fix and lyses mixture were added, the tubes were vortexed immediately for one second and incubation of the tube was done again for 10 minutes in dark at room temperature. Centrifugation of the tubes at low speed was done for 5 minutes followed by aspiration of supernatant and resuspension of pellet in residual fluid. Two $\mathrm{ml}$ of phosphate buffer saline (PBS) were added to each tube, the suspension was centrifuged at low speed. The supernatant was discarded, and then the residual suspension was passed through the flow cytometer. Monoclonal antibodies for TLR2 and CD14 were supplied by R \& D systems Inc (Minneopolis, Minnesota, USA). Data acquisition and analysis were performed on cell quest program of the coulter EPICS XL flow cytometry. Gating on monocytes, 10000 events were acquired and statistical analysis was done by cell quest software. Results expressed as mean florescence intensity (MFI).

\section{Results}

\section{Descriptive statistics}

IL-18 levels were significantly higher in obese non-diabetic group and obese diabetic group when compare to normal control group $(\mathrm{p}<0.001)$ (Table 1 and Figure 1$)$.

Also there was a highly significant increase in TLR2 MFI in obese non-diabetic group, obese diabetic group when compared to controls group $(\mathrm{p}<0.001)$, also levels of TLR2 MFI were significantly higher in obese diabetic group compared to obese non-diabetic group $(\mathrm{p}<0.001)$ (Table 2 and Figure 1).

There was a significant increase in HOMA-IR in obese non-diabetic group and a high significant increase diabetic in obese group when compared to controls group $(\mathrm{p}<0.05)(\mathrm{P}<0.001)$ respectively (Table 2 and Figure 2).

\begin{tabular}{|c|c|c|c|c|c|c|}
\hline & $\begin{array}{l}\begin{array}{l}\text { Obese non } \\
\text { diabetic } \\
\mathrm{N}=15\end{array}\end{array}$ & $\begin{array}{l}\text { Diabetic obese } \\
N=15\end{array}$ & $\begin{array}{l}\text { Control } \\
N=10\end{array}$ & P1 & $\mathbf{P 2}$ & P3 \\
\hline & \multicolumn{3}{|l|}{ Mean \pm SD } & $\begin{array}{l}0.7 \\
13\end{array}$ & $\begin{array}{l}0 . .0 \\
00\end{array}$ & 0.0 \\
\hline IL-18(ng/I) & $61.5+8.2$ & $62.8+10.9$ & $\begin{array}{l}26.5+ \\
6.5\end{array}$ & & & 00 \\
\hline TLR2 MIF & $9.3+3.2$ & $16.7+3.4$ & $\begin{array}{l}4.5 \\
1.2\end{array}$ & $\begin{array}{l}0.0 \\
0^{\star *}\end{array}$ & $\begin{array}{l}0 . .0 \\
00\end{array}$ & $\begin{array}{l}0 . \\
00 \\
0\end{array}$ \\
\hline HOMA-IR & $3.81+0.97$ & $11.55+3.89$ & $\begin{array}{l}2.46 \\
0.95\end{array}+$ & $\begin{array}{l}0.0 \\
0^{\star *}\end{array}$ & $\begin{array}{l}0.0 \\
025\end{array}$ & $\begin{array}{l}0 . \\
00 \\
* *\end{array}$ \\
\hline Insulin $(\mu \mathrm{IU} / \mathrm{ml})$ & $16.4+3.72$ & $21.40+3.98$ & $\begin{array}{l}11.2 \\
2.94\end{array}+$ & $\begin{array}{l}0.0 \\
014\end{array}$ & $\begin{array}{l}0.0 \\
08\end{array}$ & $\begin{array}{l}0 . \\
00\end{array}$ \\
\hline
\end{tabular}

Table 2: Comparison between IL18, TLR 2 MFI, HOMA-IR and INSULIN in obese non diabetic, obese diabetic patients and healthy controls.

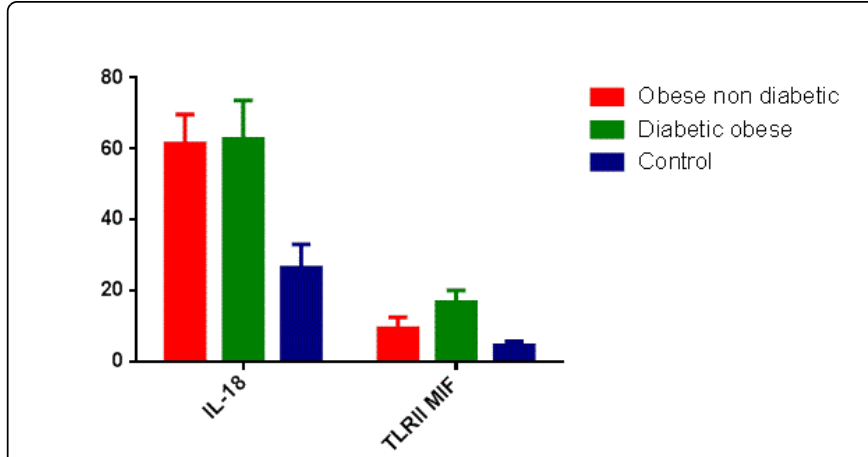

Figure 1: Level of IL18 and TLR2 in obese, diabetic obese and control groups.

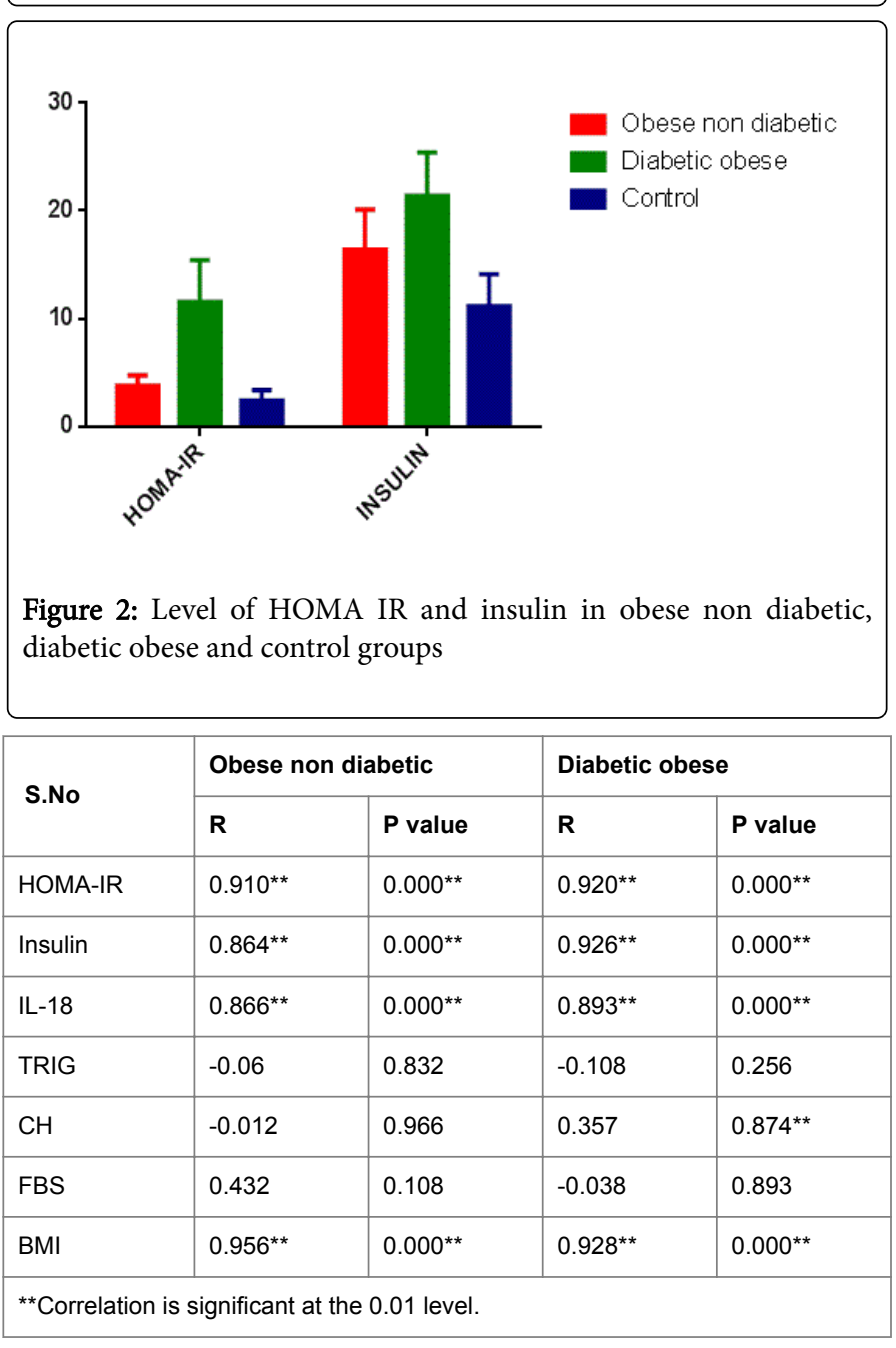

Table 3: Correlation of TLR2MFI with various laboratory parameters of all groups. 


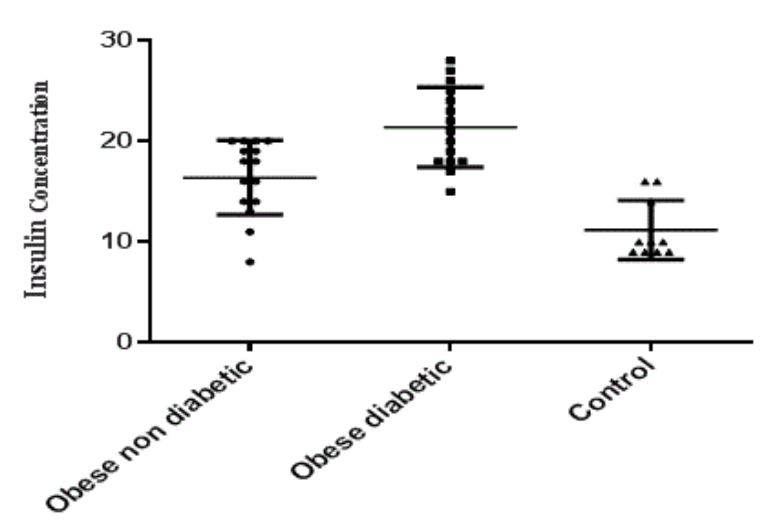

Groups

Figure 3: Insulin in obese non diabetic, diabetic obese and control groups.

There was a significant increase in insulin levels in obese nondiabetic group, obese diabetic group when compared to controls group $(p<0.05)$, also levels of insulin were significantly higher in obese nondiabetic group compared to obese diabetic group $(\mathrm{p}<0.05)$ (Table 2 and Figure 2). Also levels of HOMA IR were significantly higher in obese diabetic group compared to obese non-diabetic group $(\mathrm{p}<0.001)$ (Figure 2).

As regard correlation study, Table 3 and Figure 3 shows significant positive correlation between TLR2 and each of Insulin, IL18 and BMI in obese non-diabetic and obese diabetic groups and with HOMA IR in obese diabetic group $(\mathrm{p}<0.001)$.

\section{Discussion}

Obesity has become a worldwide health problem because it is strongly associated with metabolic syndromes including type 2 diabetes mellitus (T2DM), atherosclerosis and ischemic heart diseases. Accumulating evidence indicates that chronic-low grade inflammation has a crucial role in the pathogenesis of obesity-related metabolic dysfunction [10].

The chronic inflammatory alternations are associated with dynamic changes in the composition and function of immune cells in various tissues such as adipose tissue, pancreatic islet, liver, muscle, and hypothalamus, Chronic systemic inflammation is considered to play an important role in the development of diabetes and it may represent a unifying link between metabolic syndrome, type 2 diabetes, and cardiovascular disease [11] (Figure 4).

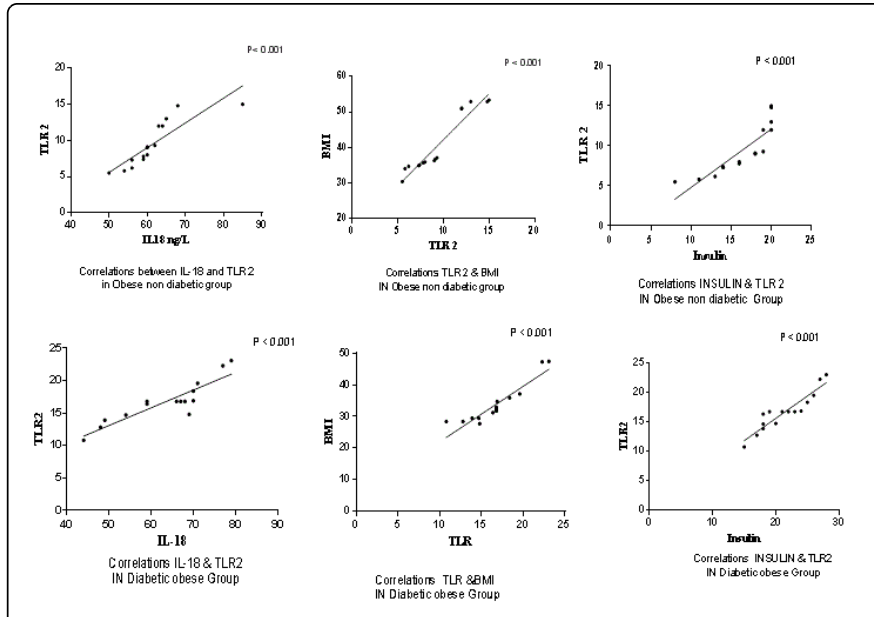

Figure 4: Graphical representation.

Type 2 diabetes mellitus is a metabolic and inflammatory disease characterized by deteriorating $\beta$-cell function and increased levels of inflammatory cytokines. Low-grade inflammation and innate immune system activation lead to $\beta$-cell failure. Obesity-associated chronic tissue inflammation is a key contributing factor to type 2 diabetes mellitus, and a number of studies have clearly demonstrated that the immune system and metabolism are highly integrated. Advances in deciphering the various immune cells and signaling networks that link the immune and metabolic systems have contributed to understanding the pathogenesis of obesity-associated inflammation. Other studies have suggested that pattern recognition receptors in the innate immune system recognize various kinds of endogenous and exogenous ligands, and have a crucial role in initiating or promoting obesityassociated chronic inflammation. Importantly, these mediators act on insulin target cells or on insulin-producing cells impairing insulin sensitivity and its secretion [14].

TLRs are mostly expressed in innate immune cells, such as macrophages and dendritic cells. Among the TLR family members, TLR2 and TLR4 have emerged as important regulators of metabolic inflammation. TLR 2 and TLR4 are broadly expressed in adipocytes, hepatocytes, myocytes and in the hypothalamus and islets. Their expression is increased in muscle and adipose tissues of obese and obese diabetic patients and in circulating monocytes of patients with T2D, which correlates with the severity of insulin resistance [14].

This study was conducted to determine the role of TLR2 in the pathogenesis of type 2 diabetes in obese patient. Expression of TLR2 in peripheral blood monocytes was significantly elevated in obese nondiabetic and obese diabetic patient compared to control group $(\mathrm{p}<0.001)$, this expression was positively correlated with insulin and BMI in obese non-diabetic and obese diabetic and with HOMA IR in obese diabetic $(\mathrm{p}<0.001)$.

These results are consistent with the previous studies of Dasu and Devaraj et al. who revealed that type 2 diabetic subjects had significantly increased TLR-2, TLR4 mRNA, and protein in monocytes compared with control subjects $(P<0.05)$ and Increased TLR2 and TLR4 expression was correlated with BMI, homeostasis model assessment-insulin resistance (HOMA-IR), glucose, A1C, Ne(carboxymethyl) lysine (CML), and free fatty acid (FFA). 
Ahmad, R et al (2012) (16) also found, significantly increased expression of TLR2 and TLR4 in both peripheral blood mononuclear cells (PBMCs) and subcutaneous adipose tissue (SAT ) of obese and overweight subjects compared to thin controls. And There was significant correlation of expression of TLR2 and TLR4with body mass index (BMI). our results also are consistent with (Andrews, $S$ et al 2015)(17)who revealed increased gene expression of TNF- $\alpha$, IL-6, NF$\kappa \mathrm{B}$, and TLR-2/4 in patients with diabetes, obesity, or both exacerbate and perpetuate the insulin resistance and inflammatory state.

This could be explained by the fact that Toll-like receptors (TLRs) are highly conserved transmembrane receptors with widespread expression on the cells most involved in the pathogenesis of metabolic syndrome, namely adipocytes and macrophages. Each TLR recognizes a specific repertoire of ligands and is capable of initiating activation of $\mathrm{NF}-\kappa \mathrm{B}$, a master regulator of the molecular inflammatory response. Though classically described as receptors for antigens associated with a variety of bacteria, viruses, and fungi, recent reports suggest that TLRs also sense and respond to nutritional fatty acids. In this capacity, TLRs may be viewed as a possible bridge between nutrition and molecular inflammation, the harbinger of metabolic syndrome [15-18].

IL-18 is proinflammatory cytokine and has been suggested to be produced by adipose tissue. Circulating IL-18 levels have been shown to be increased in obese subjects and reduced with weight loss. Moreover, overexpression of IL-18 aggravated insulin resistance in a rat model of metabolic syndrome. A lack of IL-18 or its receptor in mice induces hyperplasia, obesity and insulin resistance [19].

Additionally, high circulating levels of IL-18 are associated with an increased risk of IR. In apparently healthy non obese persons, high IL-18 levels are associated with future diabetes incidence. This association was independent of well-known risk factors for diabetes, including obesity and dietary intake [20,21].

The present study demonstrated that obese diabetic and nondiabetic subjects had significantly higher serumIL-18 levels than lean individuals $(\mathrm{p}<0.001)$ and its levels were correlated with INSULIN AND BMI in both subjects and correlated with HOMA IR in group in obese diabetic patients $(\mathrm{p}<0.001)$. Such data agrees with previous findings of Mabrouk and Ghareeb et al. who demonstrated that obese diabetic and non-diabetic subjects had significantly higher serum resisting, visfatin and IL-18 levels than lean individuals. Ahmad et al. [22] suggested that concentrations of OPN and IL-18 cytokines were simultaneously increased in obese individuals as compared with lean individuals and the levels correlated positively to each other. In addition, Circulating levels of IL-18 are elevated in obesity and correlate with body mass index, adiposity and insulin resistance. These results could be attributed to that IL-18 acts in synergy with IL-12 to stimulate Th1 polarization, and levels of IL-12 have been reported to be increased in subjects with type 2 diabetes. Hence, it could be speculated that IL-18 in combination with a hyperglycemic proinflammatory milieu might trigger Th1 activation, which was associated with IR [23]. Additionally, polymorphisms in the IL-18 gene had been shown to be associated with increased serum levels of IL-18, impaired insulin sensitivity and increased risk of having the metabolic syndrome [23].

Furthermore, it was shown that patients with obesity and type 2 diabetes, or even apparent healthy non obese persons (who have insulin resistance) produce significantly less IFN- $\gamma$ from peripheral blood mononuclear cells (Th1) in response to IL-18 stimulation compared to lean healthy controls, most likely due to reduced expression of the IL-18 receptor $\beta$ chain, so IL-18 resistance is a potential explanation of elevated IL-18 levels in such patients [24].

On the other hand, another group of researchers reported no differences in IL-18 levels between obese and lean subjects [25-28]. The reasons for such conflicting results may be due to ethnic heterogeneity, different population characteristics such as gender and age or confounding factors.

The current study, showed a significant positive correlation between TLR2 MFI and IL18 levels in obese diabetic and obese non-diabetic subjects. These results could be attributed to that TLRs can be activated by a variety of dietary factors and endogenous damage-associated signals (DAMPs) in response to obesity-induced metabolic stress. Both TLR2 and TLR4 have been shown to sense free fatty acids; in addition, ceramides, high-mobility group box 1(HMGB1), fetuin-A, heat shock proteins, and modified LDLs can also activate TLR4. TLR2 and TLR4 then signal through MyD88 to activate the NF- $\kappa B$ and MAPK pathways to inhibit insulin signaling through insulin receptor substrate (IRS) serine phosphorylation and to induce the transcription of proinflammatory cytokines, such as TNF and IL-6, as well as proIL-1 $\beta$ and proIL-18 [24].

\section{Conclusions}

This study demonstrated that the expression of TLR-2 on $\mathrm{CD} 14+$ monocytes was up regulated in obese diabetic patients and was associated with inflammatory response as assessed by increased serum levels of IL-18. These findings indicated that TLR-2, and IL-18 played a significant role in pathogenesis of type 2 diabetes in obese patient. Which could be used to pave the way for prophylactic or therapeutic procedures?

\section{Conflict of Interest}

The authors declare no conflict of interest. This research received no specific grant from any funding agency in the public or commercial sphere.

\section{References}

1. Mozumdar, A. and Liguori G (2011). "Persistent increase of prevalence of metabolic syndrome among US adults: NHANES III to NHANES 19992006." Diabetes care34(1): 216-219.

2. Gatineau Mary, Hancock Caroline, Holman Naomi, Outhwaite Helen, Oldridge Lorraine, Christie Anna and Ells Louisa (2014)Adult obesity and type 2 diabetes July, PHE publications gateway number: 2014211

3. Könner AC, Brüning JC(2011), Toll-like receptors: linking inflammation to metabolism. Trends Endocrinol Metab 2011: 22(1):16-23.

4. Dasu, M. R., S. Devaraj, L. Zhao, . Hwang D.H and. Jialal .I (2008). "High glucose induces toll-like receptor expression in human monocytes Mechanism of activation." Diabetes57(11): 3090-3098.

5. Curtiss, L. K. and Tobias ,P.S (2009). "Emerging role of Toll-like receptors in atherosclerosis." Journal of lipid research50(Supplement): S340-S345.

6. Bagarolli, R. A., Saad ,M.J.A and Saad ,S.T.O(2010). "Toll-like receptor 4 and inducible nitric oxide synthase gene polymorphisms are associated with type 2 diabetes." Journal of Diabetes and its Complications24(3): 192-198.

7. Jialal, I. and H. Kaur (2012). "The role of toll-like receptors in diabetesinduced inflammation: implications for vascular complications." Current diabetes reports12(2): 172-179.

8. Skurk, T., H. Kolb, S. Müller-Scholze, K. Röhrig, H. Hauner and C. Herder (2005). "The proatherogenic cytokine interleukin-18 is secreted by human adipocytes." European Journal of Endocrinology152(6): 863-868. 
Citation: Mohamed ZA, Abd Elraheem SE, EL Nahrery EM, Sabry S, Mohamed MSE (2016) TLR2 and IL 18 in Obese Diabetic Patient. J

9. Dinarello, C. A. (2006). "Interleukin 1 and interleukin 18 as mediators of inflammation and the aging process." The American journal of clinical nutrition83(2): 447S-455S

10. Presta I, Andreozzi F, Succurro E, Marini MA, Laratta E, Lauro R, et al. (2009): IL-18 gene polymorphism and metabolic syndrome. Nutr Metab Cardiovasc Dis, 19:e5-e6.

11. Kumar, R. A., K. Narasimhasetty, H. Murthy, S. Somannavar, L. Murali, P. V. Bhende, R. Lalitha and S. B. Shetty (2013). "Comparison of insulin resistance scoring system with other indirect methods of estimating insulin resistance in south Indian type 2 diabetes individuals."

12. Osborn, O. and Olefsky ,J.M (2012). "The cellular and signaling networks linking the immune system and metabolism in disease." Nature medicine18(3): 363-374.

13. Ryan, K. K., S. C. Woods and Seeley ,R.J (2012). "Central nervous system mechanisms linking the consumption of palatable high-fat diets to the defense of greater adiposity." Cell metabolism15(2): 137-149.

14. Watanabe, Y., Y. Nagai and Takatsu ,K (2013). "Activation and regulation of the pattern recognition receptors in obesity-induced adipose tissue inflammation and insulin resistance." Nutrients5(9): 3757-3778.

15. Dasu, M. R., S. Devaraj, S. Park and Jialal .J (2010). "Increased toll-like receptor (TLR) activation and TLR ligands in recently diagnosed type 2 diabetic subjects." Diabetes care33(4): 861-868.

16. Ahmad, R., A. Al-Mass, V. Atizado, A. Al-Hubail, F. Al-Ghimlas, M. AlArouj, A. Bennakhi, S. Dermime and K. Behbehani (2012). "Elevated expression of the toll like receptors 2 and 4 in obese individuals: its significance for obesity-induced inflammation." Journal of inflammation 9(1): 1-11.

17. Andrews, M., N. Soto and M. Arredondo-Olguín (2015). "Association between ferritin and hepcidin levels and inflammatory status in patients with type 2 diabetes mellitus and obesity." Nutrition31(1): 51-57.

18. Jialal, I., H. Kaur and Devaraj.S (2013). "Toll-like receptor status in obesity and metabolic syndrome: a translational perspective." The Journal of Clinical Endocrinology \& Metabolism99(1): 39-48.
19. Un Ju Jung and Myung-S (2014)Obesity and Its Metabolic Complications: The Role of Adipokines and the Relationship between Obesity, Inflammation, Insulin Resistance, Dyslipidemia and Nonalcoholic Fatty Liver DiseaseInt J Mol Sci.Apr; 15(4): 6184-62.

20. Hivert, M., Q. Sun, P. Shrader, C. Mantzoros, J. Meigs and Hu .F(2009). "Circulating IL-18 and the risk of type 2 diabetes in women." Diabetologia52(10): 2101-2108.

21. Mabrouk, R., H. Ghareeb, A. Shehab, K. Omar, R. H. El-Kabarity, D. A. Soliman and Mohamed,N.A (2013). "Serum visfatin, resistin and IL-18 in a group of Egyptian obese diabetic and non diabetic individuals." Egypt J Immunol20(1): 1-11

22. Ahmad, R., A. Al-Mass, D. Al-Ghawas, N. Shareif, N. Zghoul, M Melhem, A. Hasan, F. Al-Ghimlas, S. Dermime and Behbehani.K (2013). "Interaction of osteopontin with IL-18 in obese individuals: implications for insulin resistance." PloS one8(5): e63944.

23. Wegner, M., H. Winiarska, T. Bobkiewicz-Ko and Dworacka.M (2008). "IL-12 serum levels in patients with type 2 diabetes treated with sulphonylureas." Cytokine42(3): 312-316.

24. Troseid, M., I. Seljeflot and Arnesen.H (2010). "The role of interleukin-18 in the metabolic syndrome." Cardiovasc Diabetol9(11).

25. Leick, L., B. Lindegaard, D. Stensvold, P. Plomgaard, B. Saltin and Pilegaard ,H (2007). "Adipose Tissue Interleukin-18 mRNA and Plasma Interleukin-18: Effect of Obesity and Exercise." Obesity15(2): 356-363.

26. Hofsø, D., T. Ueland, H. Hager, T. Jenssen, J. Bollerslev, K. Godang, P. Aukrust, J. Røislien and Hjelmesæth ,J (2009). "Inflammatory mediators in morbidly obese subjects: associations with glucose abnormalities and changes after oral glucose." European Journal of Endocrinology161(3): 451-458.

27. Jin, C. and R. Flavell ,R.A (2013). "Innate sensors of pathogen and stress: linking inflammation to obesity." Journal of Allergy and Clinical Immunology132(2): 287-294. 